\title{
Partitioning into Colloidal Structures of Fasted State Intestinal Fluid Studied by Molecular Dynamics Simulations
}

\author{
Michael Holmboe, ${ }^{\dagger, \dagger}$ Per Larsson, ${ }^{\dagger}$ Jamshed Anwar, ${ }^{\S}$ and Christel A. S. Bergström ${ }^{*}{ }^{\dagger}$ \\ ${ }^{\dagger}$ Department of Pharmacy, Uppsala University, Uppsala Biomedical Centre, P.O. Box 580, SE-751 23 Uppsala, Sweden \\ ${ }^{\star}$ Department of Chemistry, Umeå University, Umeå, Sweden \\ ${ }^{\S}$ Chemical Theory \& Computation, Department of Chemistry, University of Lancaster, Lancaster LA1 4YB, U.K.
}

\section{Supporting Information}

\begin{abstract}
We performed molecular dynamics (MD) simulations to obtain insights into the structure and molecular interactions of colloidal structures present in fasted state intestinal fluid. Drug partitioning and interaction were studied with a mixed system of the bile salt taurocholate $(\mathrm{TCH})$ and 1,2-dilinoleoyl-sn-glycero-3-phosphocholine (DLiPC). Spontaneous aggregation of TCH and DLiPC from unconstrained MD simulations at the united-atom level using the Berger/ Gromos54A7 force fields demonstrated that intermolecular hydrogen bonding between TCH molecules was an important factor in determining the overall $\mathrm{TCH}$ and DLiPC configuration. In bilayered systems, these intermolecular

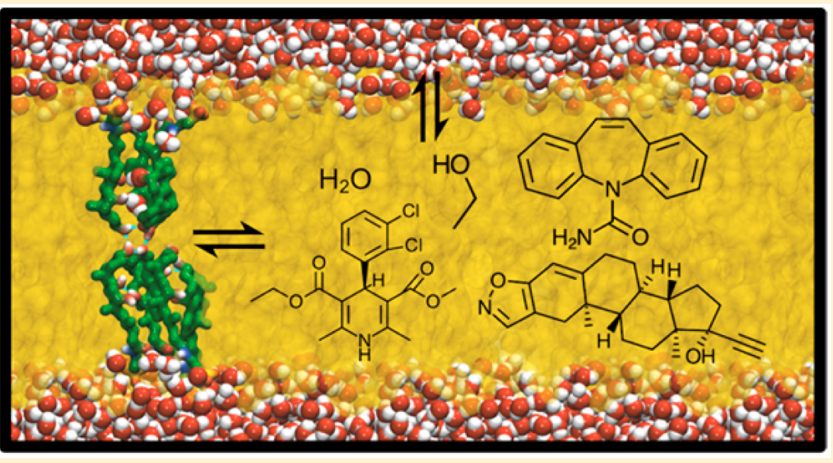
hydrogen bonds resulted in embedded transmembrane $\mathrm{TCH}$ clusters. Free energy simulations using the umbrella sampling technique revealed that the stability of these transmembrane $\mathrm{TCH}$ clusters was superior when they consisted of 3 or $4 \mathrm{TCH}$ per bilayer leaflet. All-atom simulations using the Slipids/GAFF force fields showed that the TCH embedded in the bilayer decreased the energy barrier to penetrate the bilayer $\left(\Delta G_{\text {pen }}\right)$ for water, ethanol, and carbamazepine, but not for the more lipophilic felodipine and danazol. This suggests that diffusion of hydrophilic to moderately lipophilic molecules through the bilayer is facilitated by the embedded TCH molecules. However, the effect of embedded TCH on the overall lipid/water partitioning was significant for danazol, indicating that the incorporation of TCH plays a crucial role for the partitioning of lipophilic solutes into e.g. lipidic vesicles existing in fasted state intestinal fluids. To conclude, the MD simulations revealed important intermolecular interactions in lipidic bilayers, both between the bile components themselves and with the drug molecules.
\end{abstract}

\section{INTRODUCTION}

The hunt for highly potent new drug molecules, often targeting receptors with lipophilic molecular requirements, has resulted in a general trend of increasing number of highly lipophilic, poorly water-soluble drugs. ${ }^{1-3}$ Between 75 and $90 \%$ of all new drug molecules have a solubility which is too low to allow complete dissolution of the dose in the intestinal fluid after oral administration. ${ }^{4,5}$ Therefore, the bioavailability of the drug is compromised due to low and erratic absorption. In the gastrointestinal tract lipophilic drug molecules are solubilized in mixed lipid aggregates in the intestinal fluids. In the fasted state, these are composed of bile salts and phospholipids. For lipophilic drug molecules, the interaction of the drug with the bile salts and phospholipids can have a significant effect on the partitioning into these structures and, hence, impact the solubilization and, consequently, the bioavailability. The composition of the intestinal fluid shows high interindividual variability in the fasted state, ${ }^{6,7}$ and the large variation in bile secretion and composition adds another unpredictable element to drug delivery. Unpredictable bioavailability can be critical when the drug has a limited therapeutic window; i.e., a low dose may be ineffective while a high dose results in toxicity.

The two primary components in simulated human intestinal fluid are the bile salt taurocholate $(\mathrm{TCH})$ and the phospholipid phosphatidylcholine 1,2-dilinoleoyl-sn-glycero-3-phosphocholine (DLiPC) ${ }^{8,9}$ Phospholipids have polar headgroups and long (nonpolar) acyl chains (Figure 1). They therefore tend to self-assemble into ordered and extended bilayered membranes and micelles in water to minimize the exposure of their nonpolar groups to water. Bile salts are amphiphilic surfactants that easily self-assemble into small micelles in water solutions at millimolar concentrations, ${ }^{10-16}$ with the capacity to solubilize both polar and nonpolar compounds. ${ }^{17,18}$ Bile salts originate from cholesterol in the liver and contain a hydrophilic side chain attached to a large, rigid, and boat-shaped tetracyclic ring system. The ring system is characterized by a hydrophilic and a hydrophobic face, where the overall amphiphilic properties

Received: August 11, 2016

Revised: November 4, 2016

Published: November 14, 2016 


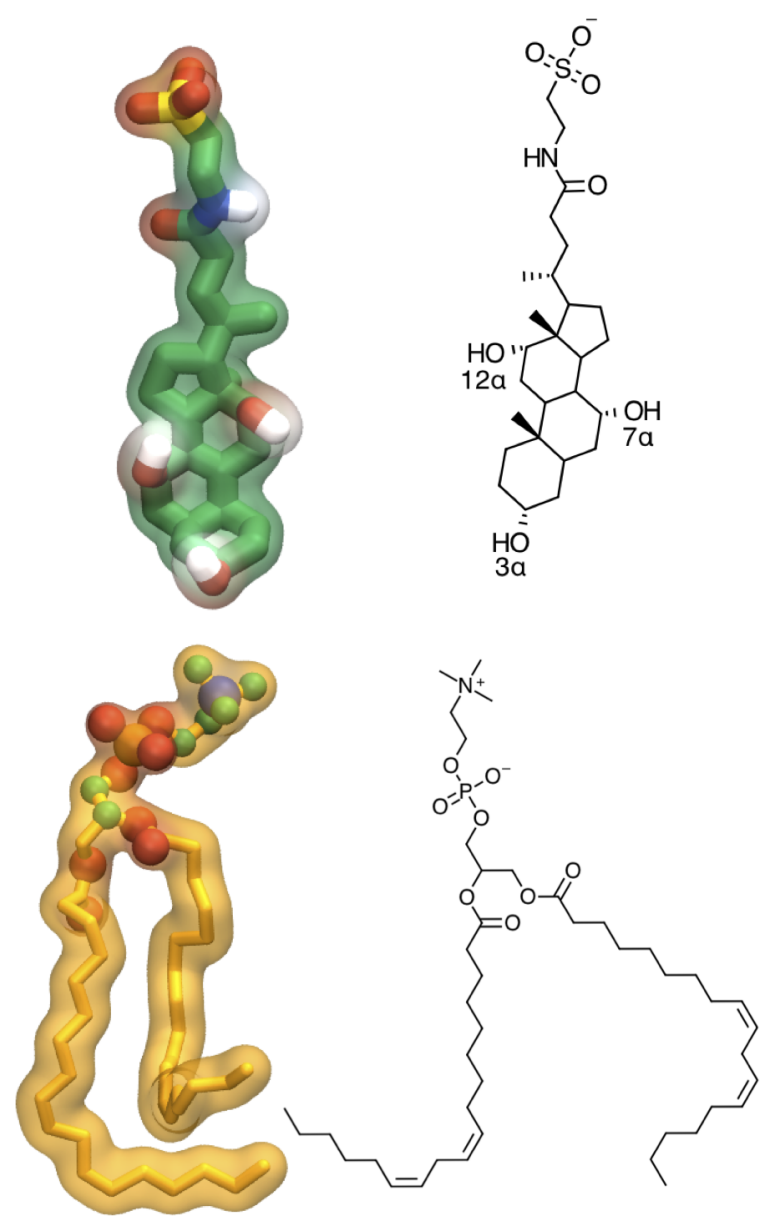

Figure 1. United-atom molecular dynamics snapshots and Lewis structures illustrating the deprotonated bile salt taurocholate $(\mathrm{TCH}$, top) and the phospholipid 1,2-dilinoleoyl-sn-glycero-3-phosphocholine (DLiPC, bottom). Note the stereochemistry of the TCH hydroxyl groups.

stem from the hydrophilic hydroxyl and hydrophobic methyl groups located on different sides of the ring system (see Figure 1). ${ }^{19}$

Experimental characterization and drug dissolution studies show that mixtures of DLiPC and $\mathrm{TCH}$ produce different mixed lipid aggregates that may increase the solubility of certain drugs by several tenfold. ${ }^{20}$ The conditions of formation of these mixed lipid aggregates and their colloidal structure (micellar, uni-multilamellar vesicles) are hence of fundamental importance for the solubilization of drug molecules in the intestine. The aggregate size and the type of the structures formed in the three-component systems of water/bile salt/phospholipid differ significantly compared to two-component systems (water/bile or water/phospholipid) that typically form micelles or vesicles. $^{11,21-25}$ In this study, we used MD simulations to study drug partitioning into colloidal structures typically found in fasted intestinal fluid. As the first step, we investigated spontaneous aggregation of mixed TCH and DLiPC systems and simulated a range of systems with different water/TCH/ $\mathrm{DLiPC}$ ratios on the united-atom (UA) level, starting from either random or ordered bilayer structures. Then, to quantify and further investigate the mixed lipid aggregates, we performed simulations of embedded TCH clusters in DLiPC bilayers using free energy calculations via umbrella sampling (US) simulations. Finally, we investigated the free energy barriers of the interaction of selected model drug compounds with representative DLiPC bilayer membranes, either without or in the presence of embedded TCH at a TCH:DLiPC ratio of $1: 2$, using US simulations on the all-atom level.

\section{RESULTS AND DISCUSSION}

Interaction between Taurocholate and Phospholipids. Initially, several different attempts to probe the tendency for $\mathrm{TCH}$ and DLiPC molecules to spontaneously aggregate into ordered bilayers were performed on the united-atom level using the Berger/Gromos54A7 force fields by simulating systems having random initial configurations and different $\mathrm{TCH}$ and DLiPC compositions and sizes. These initial simulations were performed in view of the findings by Marrink et al., who showed that several phospholipids (1,2-dipalmitoyl$s n$-glycero-3-phosphocholine (DPPC), 1-palmitoyl-2-oleoyl-snglycero-3-phosphocholine (POPC), 1,2-dioleoyl-sn-glycero-3phosphocholine (DOPC), and 1,2-dioleoyl-sn-glycero-3-phosphoethanolamine (DOPE)) spontaneously aggregate into an equilibrated bilayer phase in water within a few tens of nanoseconds, using random starting configurations. ${ }^{26}$ In contrast to the reported systems and simulations, only a handful of the simulated DLiPC and mixed DLiPC/TCH systems performed in this study from random starting configurations yielded well-defined lipid aggregates after more than $100 \mathrm{~ns}$ of simulations. As for DLiPC, this can be explained by the polyunsaturated acyl chains yielding a low gel to liquid phase transition temperature $\left(T_{\mathrm{m}}\right)$ of $-57{ }^{\circ} \mathrm{C} .{ }^{27}$ For comparison, this is approximately $100{ }^{\circ} \mathrm{C}$ lower than for the saturated 16- and 18-carbon analogues, DPPC and DSPC $\left(T_{\mathrm{m}}\right.$ $=41$ and $55^{\circ} \mathrm{C}$, respectively $\left.{ }^{28}\right)$. With this in mind, it appeared that the full equilibration of the self-assembly between mixed $\mathrm{DLiPC} / \mathrm{TCH}$ systems may require much longer time scales than those used in this study and that by Marrink et al. In line with previous studies, however, ${ }^{29}$ the obtained lipid structures were found to be highly dependent on the properties of the periodic boundary conditions (PBC) and pressure control. For instance, $64 \mathrm{DLiPC}$ molecules (with or without $16 \mathrm{TCH}$ molecules saturated with 55 water molecules per lipid) did form a bilayered phase when semi-isotropic pressure control was used. However, cylindrical micelles were formed with isotropic pressure control. It is interesting to note that both types of structures can be found experimentally and are known to coexist under certain conditions. ${ }^{30-33}$

Given that fully equilibrated self-assemblies of the DLiPC/ $\mathrm{TCH}$ lipid systems typically appeared to be outside the time scale of our simulations and likely subjected to PBC artifacts, the stability of preformed bilayer structures of DLiPC with $\mathrm{TCH}$ molecules were also investigated for six systems having $128 \mathrm{DLiPC}$ molecules and TCH:DLiPC ratios of $0: 1,1: 4,1: 2$, $1: 1,2: 1$, and $4: 1$, respectively, containing 55 water molecules per lipid (i.e., fully water saturated conditions). The resulting bilayer structures displayed remarkably high stability on the time scale of the simulations. Figure 2 shows the density profiles of mixed systems during $100 \mathrm{~ns}$. Interestingly, even at a 1:1 ratio, the DLiPC sustained a seemingly normal bilayer structure with the polar headgroups extending toward the aqueous phase, well beyond the TCH polar side chain. This was further confirmed by the similar positions of the DLiPC main functional groups (choline, phosphate, glycerol) in the system with a 1:2 TCH:DLiPC ratio (Figure 2, center) as compared with the pure DLiPC system (not shown). As for $\mathrm{TCH}$ (Figure 2, bottom), most of the sterol groups are buried 

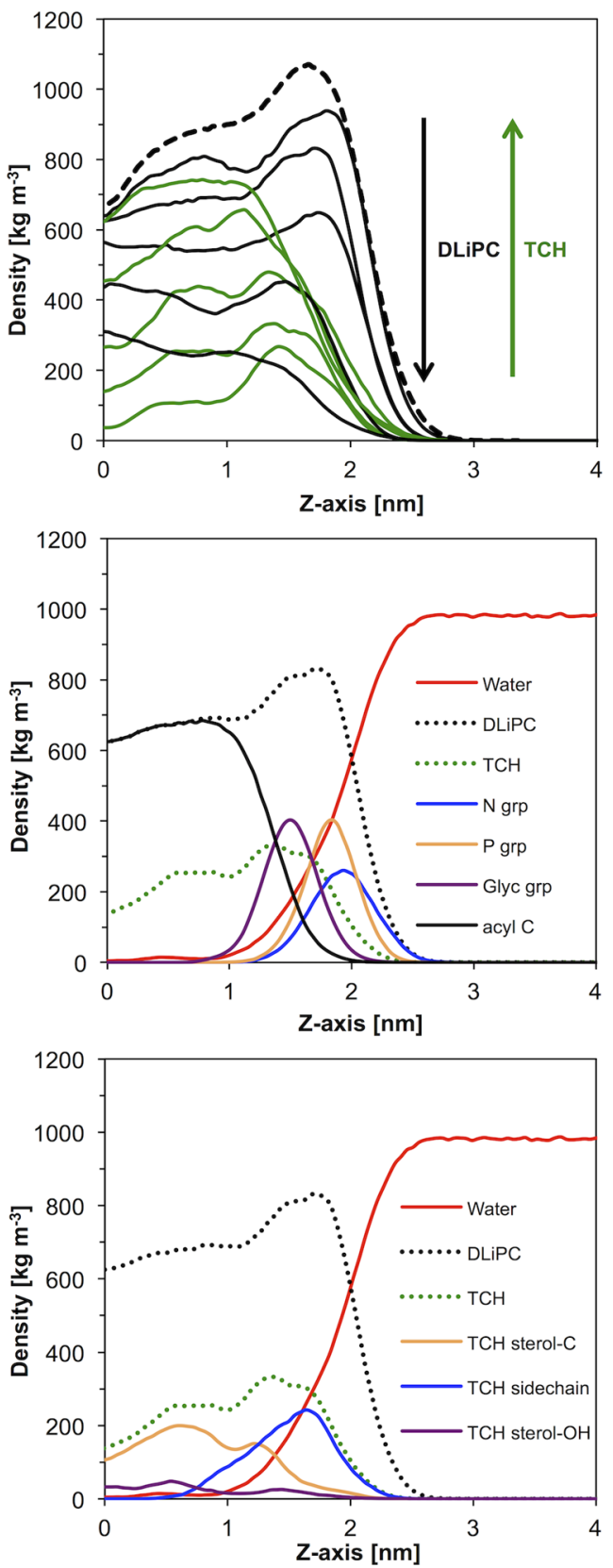

Figure 2. Top: density profiles of mixed TCH/DLiPC united-atom systems containing $0-512 \mathrm{TCH}$ (green) and $128 \mathrm{DLiPC}$ (black) molecules at ratios of $0: 1,1: 4 ; 1: 2,1: 1,2: 1$, and $4: 1 \mathrm{TCH}$ to DLiPC hydrated with 55 water molecules per lipid. Arrows indicate the effect of increasing TCH concentration. Dashed lines show the pure DLiPC system. Middle and bottom: density profiles of the 32:128 $\mathrm{TCH}: \mathrm{DLiPC}$ system, displaying the relative positions of the molecular moieties of DLiPC (middle) and TCH (bottom). For clarity, the dotted lines show the total density profiles for DLiPC and TCH. Add the following at the end: (Abbreviations in the middle panel refer to the nitrogen group ( $\mathrm{N}$ grp), the phosphate group ( $\mathrm{P}$ grp) and the glycerol linker (Glyc grp).

deep into the DLiPC acyl chains in the bilayer interior, and the polar side chains face the aqueous phase, although not beyond the DLiPC polar headgroups. Interestingly, with increasing $\mathrm{TCH}$ concentration in the simulated systems, increasing amounts of water was also found within the bilayer interior, increasing from 0 wt $\%$ to $0.2,1,1.7,3.3$, and 12 wt $\%$ with increasing TCH:DLiPC ratio.
Stability of Bilayers. The apparent stability of the mixed $\mathrm{TCH}$ :DLiPC bilayers under the given conditions might be due to the ability of the $\mathrm{TCH}$ molecules to organize into transmembrane clusters in the normal direction within the DLiPC bilayers (Figure 3). At low TCH:DLiPC ratios, most
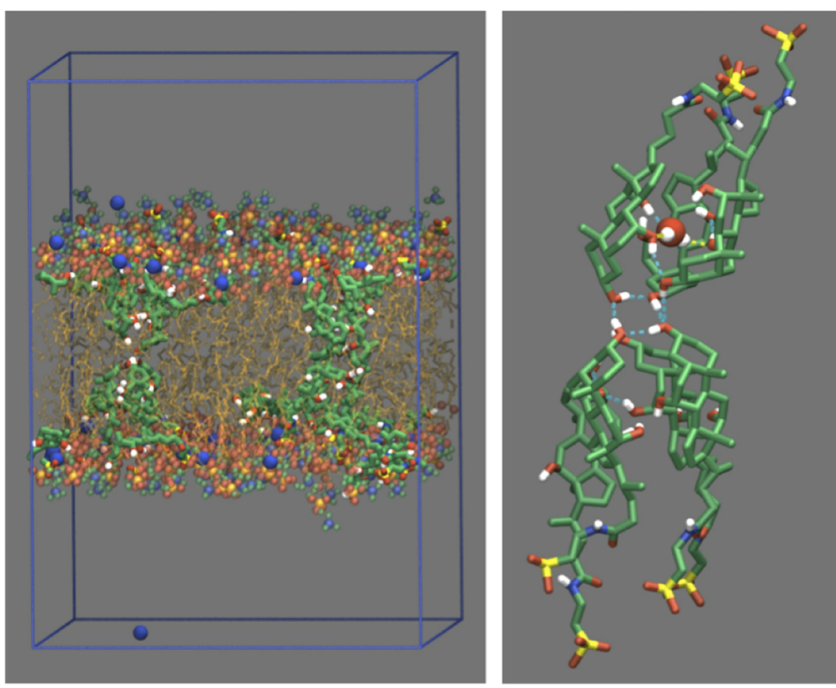

Figure 3. Left: snapshot of a mixed TCH:DLiPC united-atom bilayer system. The equilibrated structure from a bilayered system contains 32 deprotonated TCH and 128 DLiPC molecules. Water molecules are omitted for clarity; charge-balancing $\mathrm{Na}^{+}$ions are shown in blue. Right: an isolated 3/4 TCH cluster having three and four $\mathrm{TCH}$ molecules in the top and bottom bilayer leaflet, respectively. The molecules are stabilized by hydrogen bonds between the $\mathrm{TCH}$ molecules within the same bilayer leaflet and between $\mathrm{TCH}$ molecules in the opposing leaflet. Note the single water molecule in the upper $\mathrm{TCH}$ cluster; it penetrates deep into the bilayer interior due to the interaction with the buried hydrogen donors and acceptors of the TCH. PDB files with these structures are available as Supporting Information.

$\mathrm{TCH}$ molecules were found slightly below the DLiPC headgroups; the nonpolar groups faced the bilayer interior, and the polar groups interacted with the DLiPC headgroups and water molecules. However, with increasing $\mathrm{TCH}$ concentrations within the bilayer, the number of transmembrane clusters increased, extending throughout the bilayer in the normal direction. This behavior can be explained by the amphiphilic nature of $\mathrm{TCH}$, which maximizes the hydrophilic and hydrophobic interactions of the polar and nonpolar groups simultaneously.

To study the stability of the transmembrane $\mathrm{TCH}$ clusters as a function of number of TCH molecules per cluster, a set of simulations were performed with systems of $64 \mathrm{DLiPC}$ lipids containing a single isolated $\mathrm{TCH}$ cluster. One to four $\mathrm{TCH}$ molecules were kept in the upper leaflet and four in the lower one. The TCH transmembrane clusters in systems with 1 and 2 $\mathrm{TCH}$ in the upper leaflet were not stable during the timespan of the simulation, and the cluster disaggregated. These systems had a lower probability of transmembrane hydrogen bonding, in contrast to the systems with 3 or $4 \mathrm{TCH}$ upper leaflet $\mathrm{TCH}$ molecules; hence, the TCH clusters were not stabilized. With only 1 or $2 \mathrm{TCH}$ molecules in the upper leaflet, the initial hydrogen bonds with the $\mathrm{TCH}$ in the opposing bilayer leaflet were disrupted, and the $\mathrm{TCH}$ molecules positioned themselves adjacent to and below the DLiPC headgroups after a few tens 
of nanoseconds. However, the systems with 3 or $4 \mathrm{TCH}$ in the upper leaflet remained virtually unchanged even after $200 \mathrm{~ns}$, indicating superior stability over the smaller clusters. Hydrogen bond analysis (with the maximum bond length and angle between the hydrogen bond donor and acceptor being $0.35 \mathrm{~nm}$ and $30^{\circ}$ ) further revealed a slightly larger number of $\mathrm{H}$-bonds per $\mathrm{TCH}$ molecule between both $\mathrm{TCH} / \mathrm{TCH}$ and $\mathrm{TCH} /$ DLiPC for clusters with $3 \mathrm{TCH}$ compared to $4 \mathrm{TCH}$ molecules, indicating greater stability of the former type (Figure 4).

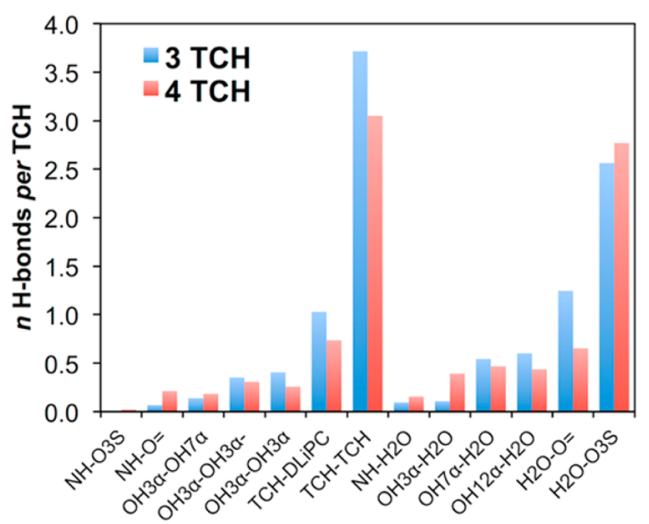

Figure 4. Hydrogen bond participation per united-atom $\mathrm{TCH}$ in transmembrane clusters containing 3 (blue) and $4 \mathrm{TCH}$ (red) molecules per bilayer leaflet. The number of inter- $\mathrm{TCH}$ hydrogen bonds (approximately 3.5) is highest in clusters with $3 \mathrm{TCH}$ molecules; in general, this number of $\mathrm{TCH}$ molecules also forms more hydrogen bonds with its surroundings.

The TCH clusters with $3 \mathrm{TCH}$ molecules in the same bilayer leaflet were often slightly tilted relative the normal direction of the bilayer, with the polar $-\mathrm{OH}$ groups in the sterol moiety facing each other. The clusters with $4 \mathrm{TCH}$ in the same bilayer leaflet typically had one peripheral and partly excluded $\mathrm{TCH}$, or one to two TCHs slightly twisted around their principal axis, exposing $-\mathrm{OH}$ groups toward the DLiPC acyl chains. Analysis of the external surface area of the entire $\mathrm{TCH}$ transmembrane clusters with a $0.14 \mathrm{~nm}$ probe (i.e., the equivalent of the water molecule radii) was also performed on the hydrophobic and hydrophilic parts of those clusters with atom charges less or greater than \pm 0.2 . This analysis revealed that the $3 \mathrm{TCH}$ clusters had an overall 15\% larger hydrophobic area and 4\% larger hydrophilic area per molecule than the clusters with 4 $\mathrm{TCH}$. However, analysis of the external surface area of the sterol moieties only (without the polar side chain) resulted in a $12 \%$ increase and $10 \%$ decrease in the hydrophobic and hydrophilic areas, respectively. This shows that the sterol groups of the $3 \mathrm{TCH}$ cluster display a more optimal packing geometry when embedded in the DLiPC bilayer than the corresponding $4 \mathrm{TCH}$ cluster, by exposing more hydrophobic and less hydrophilic atoms toward the lipid bilayer interior.

Spontaneous formation of the transmembrane $\mathrm{TCH}$ clusters was also investigated, i.e., TCH cluster formation independent of the possible artifacts introduced by using ordered starting configurations. For this, we performed simulations using a preequilibrated micelle composed of $8 \mathrm{TCH}$ embedded in the center of 64 DLiPC bilayer. Upon initial equilibration, predominately pairs of $\mathrm{TCH}$ molecules interacting with their hydrophilic groups formed within 1-2 ns, with some orienting the headgroup toward the center of the bilayer. However, during the production run $(500 \mathrm{~ns})$, a $3+3$ transmembrane $\mathrm{TCH}$ cluster formed within a few nanoseconds with the two residual $\mathrm{TCH}$ molecules positioned adjacent to the DLiPC headgroups on each side of the bilayer. This result further indicated that $\mathrm{TCH}$ transmembrane clusters could form naturally in bilayers of DLiPC.

To further investigate the TCH and DLiPC interactions in mixed lipid bilayers, the potential of mean force (PMF) for the $\mathrm{TCH}$ molecule perpendicular the bilayer ( $z$-direction) was determined using US simulations followed by the weighted histogram analysis. ${ }^{34}$ A PMF indicates the affinity of a TCH molecule for a particular position. The overall PMF profile also reveals the free energy barriers along the chosen reaction coordinate. Figure 5 shows the PMF profiles for $\mathrm{TCH}$ molecules in different transmembrane clusters over half the bilayer. For a $\mathrm{TCH}$ molecule not interacting with any other TCH molecules (denoted 1/0 in Figure 5), the PMF profile is even positive $(+8 \mathrm{~kJ} / \mathrm{mol})$ in the center of the bilayer. This clearly shows that the interior of the bilayer is an unfavorable environment for the $\mathrm{TCH}$ alone. However, with $4 \mathrm{TCH}$ molecules in the opposing bilayer leaflet (denoted as $1 / 4$ in the figure), the corresponding value decreased to $-68 \mathrm{~kJ} / \mathrm{mol}$, indicating a gain in stability by formation of a transmembrane cluster. It is also notable that the minimum of the PMF profile (the optimal position of the molecule COM) of the 1/4 system was more shifted toward the bilayer center and away from the DLiPC headgroups than the $1 / 0$ system. With additional $\mathrm{TCH}$ molecules present in the same bilayer leaflet (i.e., systems with $2 / 4$ and 3/4 $\mathrm{TCH}$ molecules in the upper and lower bilayer leaflets, respectively), there were even deeper PMF profiles, demonstrating a greater overall stability of the TCH clusters. For clusters with up to $3 \mathrm{TCH}$ molecules in the upper bilayer leaflet, the minimum of each PMF curve shifted away from the bilayer center due to the increasing interactions with adjacent $\mathrm{TCH}$. For the same systems, this trend was accompanied by an increasing free energy penetration barrier, $G_{\text {pen }}$ (defined analogously to eq 1), as indicated in Figure 5. However, the same trend was broken upon additional $\mathrm{TCH}$, suggesting that clusters with $>3 \mathrm{TCH}$ are less rigid due to the small energy gain achieved by adding an additional $\mathrm{TCH}$ to the otherwise stable and rigid $3 \mathrm{TCH}$-molecule clusters.

To better represent physiological conditions where free $\mathrm{TCH}$ molecules are also present, an additional system was created by adding $64 \mathrm{TCH}$ to the aqueous bulk phase. After equilibration, the aqueous phase TCH mostly accumulated at the DLiPC/ water interface, which drastically changed the PMF profile of the probe $\mathrm{TCH}$ molecule (Figure 5, bottom). Nevertheless, the minimum value and position of the PMF profile strongly resembled systems without bulk phase TCH. Hence, TCH was energetically favored to interact with the transmembrane $\mathrm{TCH}$ clusters in the lipid bilayer rather than interacting with the $\mathrm{TCH}$-rich water phase in contact with the DLiPC bilayer.

Drug Interactions with Bile Salt and Phospholipids. Bile salts and phospholipids such as TCH and DLiPC solubilize drugs $^{35}$ and alter lipid membrane properties. ${ }^{19}$ Therefore, additional US simulations were performed to investigate the interactions of drug molecules with the mixed lipid bilayers of DLiPC and TCH (Figure 6). The simulations were performed at the all-atom level using the Slipids and GAFF force fields, as recommended by a recent benchmarking study, ${ }^{36}$ and to better account for specific small molecule-lipid interactions. Because of the computational expense of all-atom US simulations for three-component systems, ${ }^{37-39}$ the simulations were limited to 

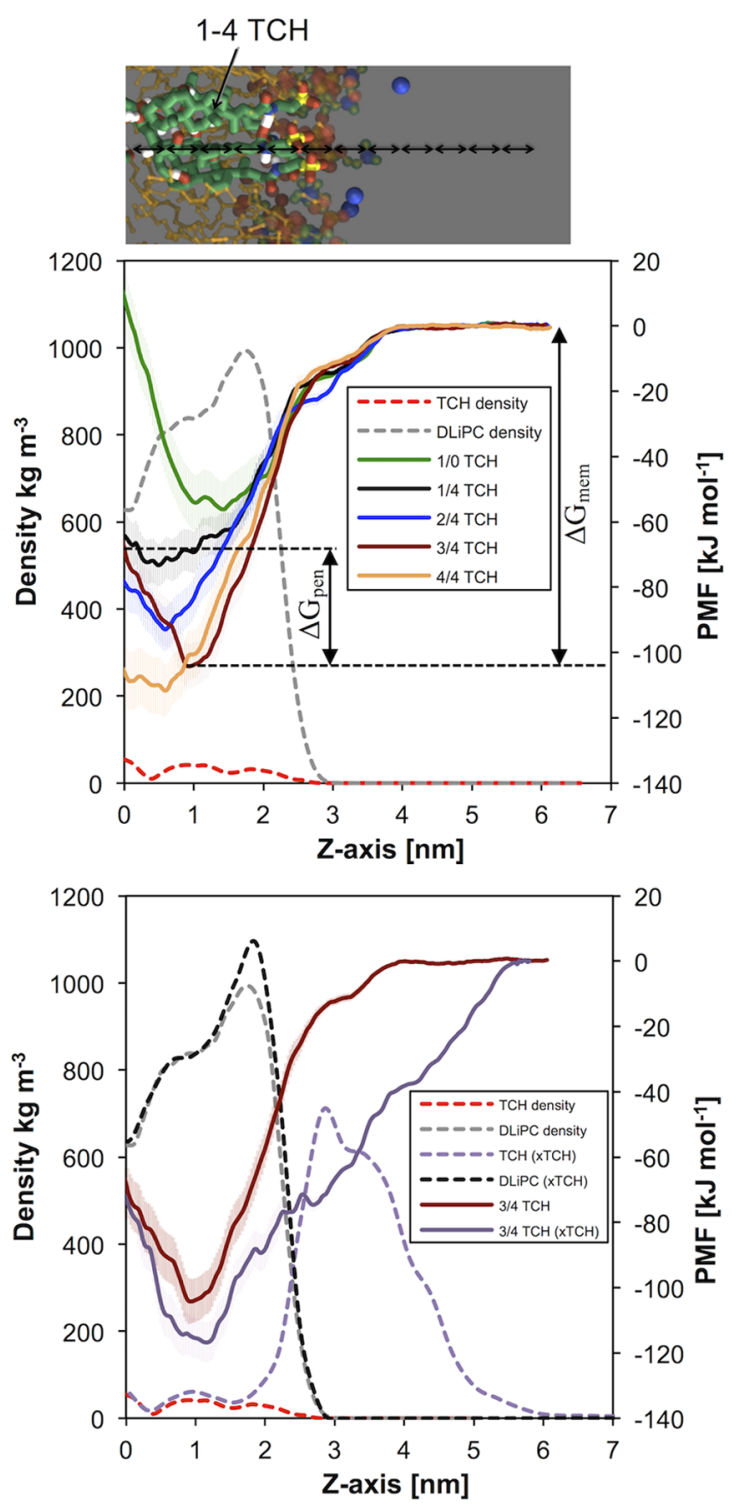

Figure 5. Density and PMF profiles for TCH/DLiPC united-atom systems. Top: simulation snapshot showing the system setup for the US simulations with black arrows indicating the approximate position of the on average 40 umbrella windows used. Middle: density profiles of DLiPC and the TCH cluster, with five separate PMF curves for $\mathrm{TCH}$ along the Z-direction, normal to the DLiPC bilayer. Note the shift in the position of the PMF minimum with a changing number of TCH molecules. Black arrows indicate $\Delta G_{\text {lipid/water }}$ and $\Delta G_{\text {pen }}$ for the $3 / 4$ cluster. Bottom: density of DLiPC and TCH and the PMF profiles for a $3 / 4 \mathrm{TCH}$ cluster without and with additional $(x \mathrm{TCH})$ of surface $\mathrm{TCH}$.

five different molecules: water, ethanol, carbamazepine, felodipine, and danazol (Figure 7). These molecules were chosen to cover a wide range of lipophilicity, and the resulting $\log P$ values, as obtained from eq 1 , are summarized in Table 1 .

For water, there was a clear decrease in the otherwise positive PMF profile in the mixed TCH/DLiPC system compared to the pure DLiPC bilayer when investigating deep partitioning into the membrane $(>1 \mathrm{~nm})$. Inspection of the trajectories revealed-in line with the results in Figure 4 and the unitedatom simulations-occasional penetration of water molecules into the transmembrane $\mathrm{TCH}$ clusters, facilitated by hydrogen bonding with the $\mathrm{TCH}$ sterol hydroxyl groups. The overall free

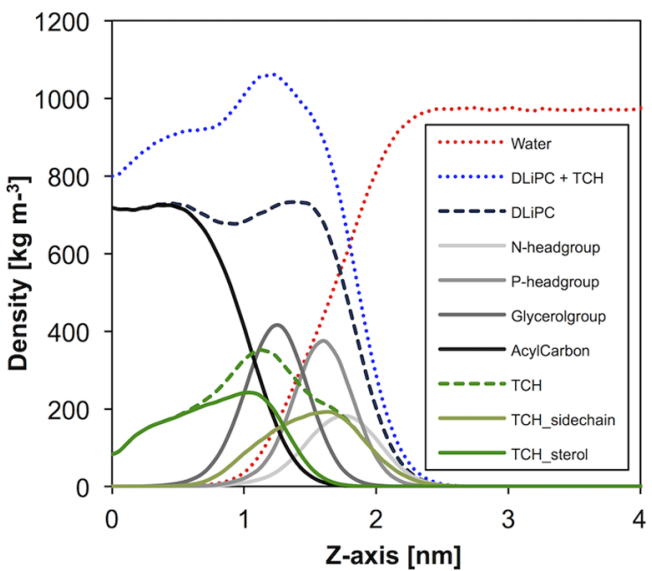

Figure 6. Density profiles of a mixed lipid bilayer containing 48 DLiPC and $24 \mathrm{TCH}$ molecules.

energy difference $\left(\Delta G_{\text {lipid/water }}\right)$ of water in the mixed TCH/ DLiPC and the DLiPC reference was $18.6 \pm 2.3$ and $27.6 \pm 0.6$ $\mathrm{kJ} / \mathrm{mol}$, respectively, well in line with previous studies. ${ }^{42}$ This demonstrates that the water partitioning of a DLiPC bilayer increases by a factor of 30 when TCH is present (calculated from $\Delta \Delta G_{\text {lipid/water }}$ and eq 1 ).

The PMF profiles over the mixed TCH/DLiPC and pure DLiPC bilayer revealed only small differences in partitioning of the drug molecules and ethanol (Table 1).

However, intricate details governing the molecular interactions between the different compounds and TCH/DLiPC were found by interaction energy analysis (separating out electrostatic and van der Waals interactions). For the lipophilic compound danazol $\left(\log P_{\text {oct }}\right.$ of 4.53), ${ }^{20}$ the corresponding $\log$ $P_{\text {lipid/water }}$ value was higher in the mixed TCH/DLiPC and the pure DLiPC systems, respectively, than that observed for octanol. The increased partitioning of danazol in the mixed $\mathrm{TCH} / \mathrm{DLiPC}$ bilayer and the shift of the minimum value in the PMF profile toward the center of the bilayer are explainable by the van der Waals interactions with the embedded $\mathrm{TCH}$ molecules; these were much stronger than the electrostatic interactions. This was further indicated by the higher total density of atoms in the mixed bilayer than in the pure bilayer (Figure 6). Despite these findings, the simulated trajectories did not point to greater extent of binding between danazol and $\mathrm{TCH}$ as compared to danazol and DLiPC.

For felodipine $\left(\log P_{\text {oct }}\right.$ of 5.58), ${ }^{20}$ the corresponding log $P_{\text {lipid/water }}$ values were lower in the mixed TCH/DLiPC than the pure DLiPC system. The decrease in partitioning of felodipine in the mixed $\mathrm{TCH} / \mathrm{DLiPC}$ bilayer resulted from both weaker van der Waals and electrostatic interactions with DLiPC. Unlike in the danazol system, these were not compensated for by the interactions with the embedded TCH. Figure $\mathrm{S} 1$ of the Supporting Information shows the overlap of the umbrella sampling windows, as well as the effect of the symmetrization procedure on the resulting PMF.

The differences in the PMF profiles for the TCH/DLiPC and pure DLiPC bilayers were smaller for the more hydrophilic carbamazepine and ethanol $\left(\log P_{\text {oct }}\right.$ values of 2.32 and -0.30 , respectively $\left.{ }^{40,41}\right)$. An inspection of the respective trajectories identified that these molecules interacted with the $\mathrm{TCH}-\mathrm{OH}$ groups in the center region, which was highly accessible due to low particle density in the bilayer center. Although the differences in the PMF profiles were small, the embedded 

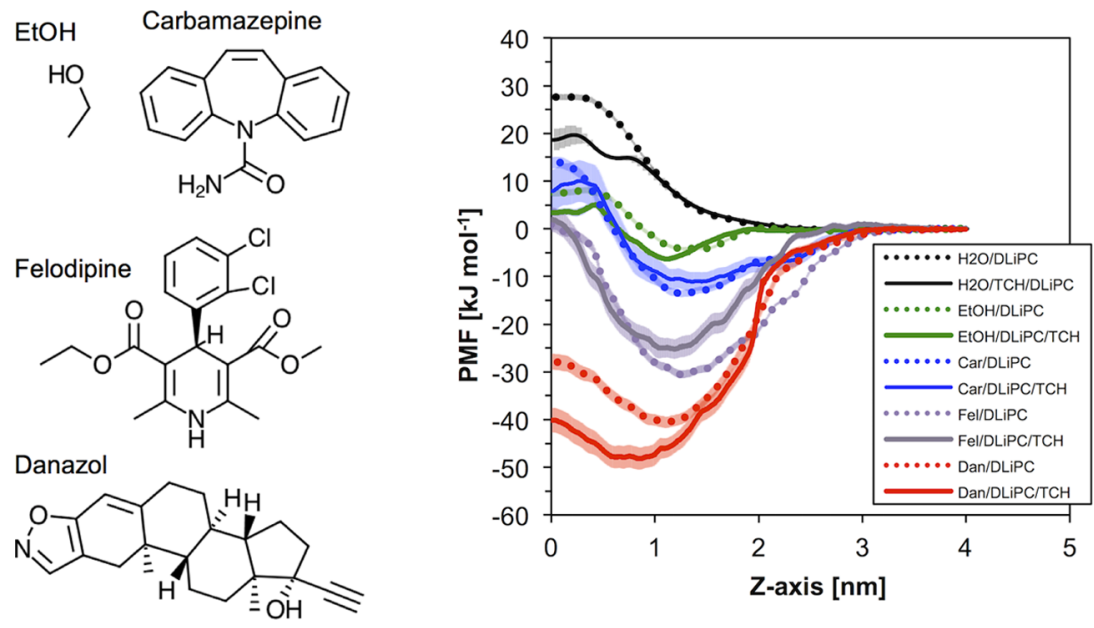

Figure 7. Left: molecular structures of ethanol (top left), carbamazepine (top right), felodipine (middle), and danazol. Right: symmetrized PMF profiles of water (red), ethanol (green), carbamazepine (blue, denoted Car), felodipine (purple, denoted Fel), and danazol (black, denoted Dan) over half an all-atom bilayer (centered at $Z=0$ ) containing only DLiPC or (dotted line) mixed TCH/DLiPC (solid line).

Table 1. The $\log P$ Values Calculated from All-Atom Simulations with Slipids/GAFF Using Eq 1

\begin{tabular}{|c|c|c|c|}
\hline & $\mathrm{TCH} / \mathrm{DLiPC}^{a}$ & $\mathrm{DLiPC}^{b}$ & $\exp ^{c}$ \\
\hline water & $-3.1 \pm 0.4$ & $-4.6 \pm 0.1$ & \\
\hline ethanol & $1.1 \pm 0.1$ & $0.75 \pm 0.05$ & $-0.3^{d}$ \\
\hline carbamazepine & $1.9 \pm 0.2$ & $2.3 \pm 0.2$ & $2.32^{e}$ \\
\hline felodipine & $4.2 \pm 0.3$ & $5.3 \pm 0.2$ & $5.58^{f}$ \\
\hline danazol & $8.1 \pm 0.5$ & $6.8 \pm 0.3$ & $4.53^{f}$ \\
\hline
\end{tabular}

${ }^{a} \log P_{\text {lipid/water }}$ in mixed TCH/DLiPC bilayer. ${ }^{b} \log P_{\text {lipid/water }}$ in pure DLiPC bilayer. ${ }^{c}$ Experimental $\log P_{\text {oct }} \cdot{ }^{d}$ Reference $40 .{ }^{e}$ Reference 41. $f_{\text {Reference } 20 .}$

TCH may accelerate drug diffusion over the lipid bilayer by lowering the $\Delta G_{\text {pen }}$. The resulting $\log P_{\text {lipid/water }}$ for carbamazepine was slightly lower in the mixed $\mathrm{TCH} / \mathrm{DLiPC}$ compared to the pure DLiPC system, which was equivalent to the reported $\log P_{\text {oct }}$ value. The corresponding $\log P_{\text {lipid/water }}$ values for ethanol were, on the other hand, slightly higher in the mixed $\mathrm{TCH} / \mathrm{DLiPC}$ compared to the pure DLiPC system; i.e., there was a slightly increased partitioning of ethanol when $\mathrm{TCH}$ was embedded.

\section{CONCLUSIONS}

The MD simulations strongly suggest that in mixtures of $\mathrm{TCH}$ and DLiPC, reflecting the composition of fasted state simulated intestinal fluid, embedded transmembrane $\mathrm{TCH}$ clusters constitute an integral component in phospholipid bilayers composed of DLiPC. This new finding complements existing models of mixed bile-phospholipid micelle and bilayer systems $^{21,23}$ by revealing the preferred number and configuration of bile salt molecules embedded in the phospholipid bilayers. Both unconstrained and US free energy united-atom simulations showed that the transmembrane $\mathrm{TCH}$ clusters were (i) stabilized by mutual hydrogen bonds $\left(\mathrm{OH}_{3 \alpha} \cdots \mathrm{OH}_{3 \alpha}\right)$ across the bilayer center and (ii) preferably consisted of 3 or $4(3+1)$ adjacent and clustered $\mathrm{TCH}$ molecules per bilayer leaflet. In the latter type, the additional $\mathrm{TCH}$ constituted a more peripheral member to the overall cluster.

From simulations on the all-atom level, the effect of embedded TCH in DLiPC bilayers on the partitioning of five model substances (water, ethanol, carbamazepine, felodipine, and danazol) showed that only water displayed a positive free energy profile over the entire bilayer regardless of embedded $\mathrm{TCH}$. For water this positive free energy barrier was however reduced in the presence of $\mathrm{TCH}$ due to penetration into the transmembrane clusters. For the other hydrophilic solutesethanol and carbamazepine-the overall lipid/water partitioning into the lipid bilayer was similar to and without embedded $\mathrm{TCH}$. The effect of embedded TCH on the overall lipid/water partitioning (i.e., from $\Delta G_{\text {lipid/water }}$ ) was most significant for danazol, demonstrating that the embedded $\mathrm{TCH}$ may facilitate partitioning into, and hence, solubilization of lipophilic solutes in the mixed bilayer. However, this was not observed for the other highly lipophilic drug felodipine, which displayed a slightly reduced $\log P_{\text {lipid/water }}$ in the presence of embedded $\mathrm{TCH}$.

\section{METHODS}

Simulation Setup and Parameters. The GROMACS ${ }^{43,44}$ suite of programs was used for all simulations. Unless otherwise stated, all lipid simulations were conducted at a physiological $\mathrm{NaCl}$ concentration of approximately $0.15 \mathrm{M}$. For the united-atom simulations (SPC/Gromos54A7/Berger ${ }^{45,46}$ ) and the all-atom simulations (Tip3P/GAFF/Slipids ${ }^{47}$ ) we used a universal short-range cutoff at $1.2 \mathrm{~nm}$ for both electrostatic and Lennard-Jones interactions. Longrange electrostatic interactions were treated by particle mesh Ewald $\left(\mathrm{PME}^{48}\right)$. A long-range dispersion correction was applied to both energy and pressure. ${ }^{49}$ All covalent bond lengths were constrained using LINCS, ${ }^{50}$ and a time step of $2 \mathrm{fs}$ was used in all production simulations.

Simulation systems of DLiPC with and without $\mathrm{TCH}$ were constructed to have either random starting configurations or ordered bilayers. The latter type was obtained by initially distributing the lipid monomers on a square grid and pre-equilibrating bilayered systems for $>50$ ns while weakly restraining the lipid movements in the direction normal to the bilayer.

DLiPC and TCH Parametrization. To model the mixed lipid systems at the united-atom level, Berger force field parameters for DLiPC were adapted from a DOPC topology, by enforcing a cisconformation of the double bonds in the acyl chains. ${ }^{45}$ For TCH, the topology and all interaction parameters were obtained from the Automated Topology Builder server using the Gromos54A7 force field. ${ }^{51}$ However, the partial charges were calculated from restricted electrostatic potential fitting using the pyRED server as OPLS compatible charges (RESP-O1), i.e., by Gaussian09 calculations at the HF/6-31G* level followed by RESP. ${ }^{52,53}$ 
Similarly, a Slipids compatible topology for DLiPC was adapted from a DOPC topology. ${ }^{54}$ This was done by removal of two hydrogen atoms and by enforcing a cis-conformation of the two neighboring double bonds and by increasing the partial charges of the $\mathrm{CH}_{2}$ atoms in the adjacent methylene group by +0.03 (to +0.06 ) to maintain charge neutrality. For the all-atom $\mathrm{TCH}$ and drug molecules, the general Amber force field (GAFF) was used, having molecular topologies constructed by Antechamber software through the acpype.py script (available online: http://code.google.com/p/ acpype/) applied to molecular coordinates obtained from the ZINC database. $^{55}$

All regular and unconstrained MD productions runs were typically preceded by (i) energy minimization using the steepest descent algorithm with a tolerance of $1000 \mathrm{~kJ} \mathrm{~mol}^{-1} \mathrm{~nm}^{-1}$, followed by (ii) a $50 \mathrm{ps}$ simulation in the canonical $(N V T)$ ensemble using position restraints on all solute particles, followed by (iii) a 5 ns volume equilibration simulation in the isothermal-isobaric (NPT) ensemble. The latter simulations used the Berendsen ${ }^{56}$ barostat for semi-isotropic pressure control, whereas all production runs used the ParrinelloRahman $^{57}$ barostat with semi-isotropic pressure coupling. For temperature control, the modified Berendsen and velocity-rescaled thermostats were used for equilibration and production runs, coupling the non-water and water molecules to separate thermostats. By analogy, removal of center of mass (COM) translation was applied to the lipids and water molecules independently; for bilayer simulations, the COM of the upper and lower bilayer leaflets were controlled separately.

Pure DLiPC and mixed TCH:DLiPC US systems-with either a random or an ordered bilayered initial configuration-were simulated for a minimum of $100 \mathrm{~ns}$, with either 64,128 , or $256 \mathrm{DLiPC}$ molecules and with a TCH:DLiPC ratio from 0 to 4:1. To ensure full water saturation, the systems contained a minimum of 30 water molecules per lipid, in contrast to 21.5 , the reported experimental value for DLiPC. 58

In the following studies we focused on free energy calculations making use of the bilayers since it is well-known that unilamellar vesicles are naturally present in fasted intestinal fluids. ${ }^{11}$ Free energy profiles for $\mathrm{TCH}$ molecules over the bilayers, i.e., the potential of mean force (PMF), were computed from US simulations. To generate the starting configurations for the sampling, a single $\mathrm{TCH}$ molecule was pulled to the aqueous bulk phase from the center of a representative $\mathrm{TCH}$ cluster embedded in a bilayer consisting of 64 DLiPC molecules. The systems contained $0-4$ adjacent TCH molecules within the same bilayer leaflet and $4 \mathrm{TCH}$ in the opposing bilayer leaflet. The total number of lipids in each leaflet was set constant by removal of DLiPC molecules from the opposing bilayer leaflet. Each starting structure was equilibrated with 90 water molecules per lipid, after which a $\mathrm{TCH}$ molecule was pulled by a harmonic force at a rate of $0.00025 \mathrm{~nm} / \mathrm{ps}$ with the Gromacs pull code. For the US simulations, more than 40 configurations were chosen from an initial set of 2500 . In these 40 configurations, the probed TCH molecule was displaced approximately $0.15 \mathrm{~nm}$ along the reaction coordinate, i.e., in the normal direction ( $z$-axis) to the bilayer. Each configuration was then simulated for $5 \mathrm{~ns}$ with a harmonic force constant of $800 \mathrm{~kJ} \mathrm{~mol}^{-1} \mathrm{~nm}^{-2}$. To ensure proper overlap of the sampled umbrella windows, each umbrella simulation was performed twice with different starting configurations. From the resulting potential of mean force (PMF) profiles an equivalent bilayer/water partitioning $\left(\log P_{\text {lipid/water }}\right)$ was calculated as

$$
\log P_{\text {lipid } / \text { water }}=\frac{\Delta G_{\text {lipid } / \text { water }}}{2.303 R T}
$$

where $\Delta G_{\text {lipid/water }}$ is the free energy of transfer from the aqueous phase to the lipid bilayer, expressed as the difference in the free energy of solubilization, $\Delta G_{\text {water }}-\Delta G_{\text {lipid }}$, i.e., analogous to calculations of the traditional octanol and water partition coefficient $\log P_{\text {oct/water }}$.

The free energy profiles over the bilayers for the small molecules (danazol, felodipine, carbamazepine, water, and ethanol) were similarly computed from US simulations, this time using all-atom systems. To evaluate the effect of $\mathrm{TCH}$ in the mixed lipid bilayers, umbrella simulations were also performed using pure DLiPC bilayers without $\mathrm{TCH}$. The mixed lipid bilayer was constructed with $24 \mathrm{TCH}$ and 48 DLiPC molecules, whereas 64 DLiPC molecules were used in the systems without TCH. Similarly to the TCH-only US simulations, representative configurations were chosen with a spacing of $0.15 \mathrm{~nm}$ along the pull vector. Each chosen configuration was simulated for 20 ns using a harmonic force constant of $700 \mathrm{~kJ} \mathrm{~mol}^{-1} \mathrm{~nm}^{-2}$. Unlike the TCH US simulations, however, 2 (or 4 for ethanol) equally spaced probed molecules were pulled simultaneously through the bilayer, i.e., sampling both sides of the bilayer leaflet at the same time. This was possible because of the small size and neutral charge of these compounds. To avoid overlap of the inserted molecules, a slow growth protocol over 50 consecutive simulation steps was applied, after which the complete drug/solvent/TCH/DLiPC structure was energy minimized and equilibrated. Because of this, a lower pull rate of $0.00005 \mathrm{~nm} / \mathrm{ps}$ was used to avoid structural artifacts in the bilayer caused by the pulled drug molecules.

To obtain the final PMF along the reaction coordinate, i.e., the free energy profiles, all US simulations were analyzed with the weighted histogram analysis method as implemented in the GROMACS g_wham utility. Standard deviations were computed from bootstrapping over 200 runs using a tolerance of $1 \times 10^{-5}$. 34 In the mixed $\mathrm{TCH} / \mathrm{DLiPC}$ system, the PMF with calculated standard deviations for water was obtained from the averaged Boltzmann-weighted density profiles of water in the drug molecule simulations, i.e., from unconstrained conditions and a total simulation time of approximately $2 \mu \mathrm{s}$.

\section{ASSOCIATED CONTENT}

\section{S Supporting Information}

The Supporting Information is available free of charge on the ACS Publications website at DOI: 10.1021/acs.langmuir.6b03008.

Table with an overview of the simulations performed as well as two additional figures with umbrella sampling plots and snapshots of TCH clusters (PDF)

Representative snapshots (PDB)

\section{AUTHOR INFORMATION}

\section{Corresponding Author}

*E-mail: christel.bergstrom@farmaci.uu.se (C.A.S.B.).

ORCID ${ }^{\circ}$

Per Larsson: 0000-0002-8418-4956

Funding

This work has received support from the European Research Council Grant 638965, the Swedish Research Council Grant 2014-3309, and the Innovative Medicines Initiative Joint Undertaking under Grant agreement no. 115369, resources of which are composed of financial contributions from the European Union's Seventh Framework Programme (FP7/ 2007-2013) and EFPIA companies. The simulations were performed on resources provided by the Swedish National Infrastructure for Computing (SNIC) at the PDC Centre for High Performance Computing (PDC-HPC), the National Supercomputer Center (NSC), and the High Performance Computing Center North (HPC2N).

\section{Notes}

The authors declare no competing financial interest.

All simulated systems were constructed using the Atomistic Trajectory Operations in Matlab (ATOM) scripts, available through the Matlab File Exchange (www.mathworks.com/ matlabcentral/fileexchange/). 


\section{ACKNOWLEDGMENTS}

The authors are grateful to Jonas Fagerberg, Olle Edholm, and David van der Spoel for valuable discussions when initiating this study.

\section{ABBREVIATIONS}

MD molecular dynamics; TCH taurocholate; DLiPC 1,2dilinoleoyl-sn-glycero-3-phosphocholine; US umbrella sampling.

\section{REFERENCES}

(1) Lipinski, C. A.; Lombardo, F.; Dominy, B. W.; Feeney, P. J. Experimental and Computational Approaches to Estimate Solubility and Permeability in Drug Discovery and Development Settings. Adv. Drug Delivery Rev. 2001, 46, 3-26.

(2) Leeson, P. D.; Springthorpe, B. The Influence of Drug-Like Concepts on Decision-Making in Medicinal Chemistry. Nat. Rev. Drug Discovery 2007, 6, 881-890.

(3) Hopkins, A. L.; Keserü, G. M.; Leeson, P. D.; Rees, D. C.; Reynolds, C. H. The Role of Ligand Efficiency Metrics in Drug Discovery. Nat. Rev. Drug Discovery 2014, 13, 105-121.

(4) Benet, L. Z.; Wu, C. Y.; Custodio, J. M. Predicting Drug Absorption and the Effects of Food on Oral Bioavailability; Bulletin Technique Gattefosse: 2006, 99, 9-16

(5) Di, L.; Kerns, E.; Carter, G. Drug-Like Property Concepts in Pharmaceutical Design. Curr. Pharm. Des. 2009, 15, 2184-2194.

(6) Bergström, C. A. S.; Holm, R.; Jørgensen, S. A.; Andersson, S. B. E.; Artursson, P.; Beato, S.; Borde, A.; Box, K.; Brewster, M.; Dressman, J.; Feng, K.; Halbert, G.; Kostewicz, E.; McAllister, M.; Muenster, U.; Thinnes, J.; Taylor, R.; Mullertz, A. Early Pharmaceutical Profiling to Predict Oral Drug Absorption: Current Status and Unmet Needs. Eur. J. Pharm. Sci. 2014, 57, 173-199.

(7) Riethorst, D.; Mols, R.; Duchateau, G.; Tack, J.; Brouwers, J.; Augustijns, P. Characterization of Human Duodenal Fluids in Fasted and Fed State Conditions. J. Pharm. Sci. 2016, 105, 673-681.

(8) Blank, M. L.; Nutter, L. J.; Privett, O. S. Determination of the Structure of Lecithins. Lipids 1966, 1, 132-135.

(9) Galia, E.; Nicolaides, E.; Hörter, D.; Löbenberg, R.; Reppas, C.; Dressman, J. B. Evaluation of Various Dissolution Media for Predicting in Vivo Performance of Class I and II Drugs. Pharm. Res. 1998, 15, 698-705.

(10) Carey, M. C. Micelle Formation by Bile Salts. Arch. Intern. Med. 1972, 130, 506

(11) Schurtenberger, P.; Mazer, N.; Kaenzig, W. Micelle to Vesicle Transition in Aqueous Solutions of Bile Salt and Lecithin. J. Phys. Chem. 1985, 89, 1042-1049.

(12) Marrink, S. J.; Mark, A. E. Molecular Dynamics Simulations of Mixed Micelles Modeling Human Bile. Biochemistry 2002, 41, 53755382.

(13) Warren, D. B.; Chalmers, D. K.; Hutchison, K.; Dang, W.; Pouton, C. W. Molecular Dynamics Simulations of Spontaneous Bile Salt Aggregation. Colloids Surf., A 2006, 280, 182-193.

(14) Pártay, L. B.; Sega, M.; Jedlovszky, P. Morphology of Bile Salt Micelles as Studied by Computer Simulation Methods. Langmuir 2007, 23, 12322-12328.

(15) Verde, A. V.; Frenkel, D. Simulation Study of Micelle Formation by Bile Salts. Soft Matter 2010, 6, 3815-3825.

(16) Madenci, D.; Egelhaaf, S. U. Self-Assembly in Aqueous Bile Salt Solutions. Curr. Opin. Colloid Interface Sci. 2010, 15, 109-115.

(17) Calabresi, M.; Andreozzi, P.; La Mesa, C. Supra-Molecular Association and Polymorphic Behaviour in Systems Containing Bile Acid Salts. Molecules 2007, 12, 1731-1754.

(18) Maldonado-Valderrama, J.; Wilde, P.; Macierzanka, A.; Mackie, A. The Role of Bile Salts in Digestion. Adv. Colloid Interface Sci. 2011, $165,36-46$
(19) Garidel, P.; Hildebrand, A.; Knauf, K.; Blume, A. Membranolytic Activity of Bile Salts: Influence of Biological Membrane Properties and Composition. Molecules 2007, 12, 2292-2326.

(20) Fagerberg, J. H.; Tsinman, O.; Sun, N.; Tsinman, K.; Avdeef, A.; Bergström, C. A. S. Dissolution Rate and Apparent Solubility of Poorly Soluble Drugs in Biorelevant Dissolution Media. Mol. Pharmaceutics 2010, 7, 1419-1430.

(21) Small, D. M.; Bourges, M. C.; Dervichian, D. G. The Biophysics of Lipidic Associations. Biochim. Biophys. Acta, Lipids Lipid Metab. 1966, 125, 563-580.

(22) Shankland, W. The Equilibrium and Structure of LecithinCholate Mixed Micelles. Chem. Phys. Lipids 1970, 4, 109-130.

(23) Mazer, N. A.; Benedek, G. B.; Carey, M. C. Quasielastic LightScattering Studies of Aqueous Biliary Lipid Systems. Mixed Micelle Formation in Bile Salt-Lecithin Solutions. Biochemistry 1980, 19, 601615.

(24) Rupp, C.; Steckel, H.; Müller, B. W. Mixed Micelle Formation with Phosphatidylcholines: the Influence of Surfactants with Different Molecule Structures. Int. J. Pharm. 2010, 387, 120-128.

(25) Kloefer, B.; van Hoogevest, P.; Moloney, R. Study of a Standardized Taurocholate-Lecithin Powder for Preparing the Biorelevant Media FeSSIF and FaSSIF. Dissolution Technol. 2010, $17,6$.

(26) Marrink, S. J.; Lindahl, E.; Edholm, O. Simulation of the Spontaneous Aggregation of Phospholipids Into Bilayers. J. Am. Chem. Soc. 2001, 123, 8638-8639.

(27) Kariel, N.; Davidson, E.; Keough, K. M. W. Cholesterol Does Not Remove the Gel-Liquid Crystalline Phase Transition of Phosphatidylcholines Containing Two Polyenoic Acyl Chains. Biochim. Biophys. Acta, Biomembr. 1991, 1062, 70-76.

(28) Mabrey, S.; Sturtevant, J. M. Investigation of Phase Transitions of Lipids and Lipid Mixtures by Sensitivity Differential Scanning Calorimetry. Proc. Natl. Acad. Sci. U. S. A. 1976, 73, 3862-3866.

(29) Warren, D. B.; Chalmers, D. K.; Pouton, C. W. Structure and Dynamics of Glyceride Lipid Formulations, with Propylene Glycol and Water. Mol. Pharmaceutics 2009, 6, 604-614.

(30) Egelhaaf, S. U.; Schurtenberger, P. Shape Transformations in the Lecithin-Bile Salt System: From Cylinders to Vesicles. J. Phys. Chem. 1994, 98, 8560-8573.

(31) Cohen, E.; Thurston, G. M.; Chamberlin, R. A.; Benedek, G. B.; Carey, M. C. Laser Light Scattering Evidence for a Common Wormlike Growth Structure of Mixed Micelles in Bile Salt- and Straight-Chain Detergent-Phosphatidylcholine Aqueous Systems: Relevance to the Micellar Structure of Bile. Biochemistry 1998, 37, 14798-14814.

(32) Leng, J.; Egelhaaf, S. U.; Cates, M. E. Kinetics of the Micelle-toVesicle Transition: Aqueous Lecithin-Bile Salt Mixtures. Biophys. J. 2003, 85, 1624-1646.

(33) Elsayed, M. M. A.; Cevc, G. The Vesicle-to-Micelle Transformation of Phospholipid-Cholate Mixed Aggregates: a State of the Art Analysis Including Membrane Curvature Effects. Biochim. Biophys. Acta, Biomembr. 2011, 1808, 140-153.

(34) Hub, J. S.; De Groot, B. L. g wham a Free Weighted Histogram Analysis Implementation Including Robust Error and Autocorrelation Estimates. J. Chem. Theory Comput. 2010, 6, 3713-3720.

(35) Fagerberg, J. H.; Bergström, C. A. Intestinal Solubility and Absorption of Poorly Water Soluble Compounds: Predictions, Challenges and Solutions. Ther. Delivery 2015, 6, 935-959.

(36) Paloncýová, M.; DeVane, R.; Murch, B.; Berka, K.; Otyepka, M. Amphiphilic Drug-Like Molecules Accumulate in a Membrane Below the Head Group Region. J. Phys. Chem. B 2014, 118, 1030-1039.

(37) Paloncýová, M.; Fabre, G.; DeVane, R. H.; Trouillas, P.; Berka, K.; Otyepka, M. Benchmarking of Force Fields for MoleculeMembrane Interactions. J. Chem. Theory Comput. 2014, 10, 41434151.

(38) Paloncýová, M.; Berka, K.; Otyepka, M. Convergence of Free Energy Profile of Coumarin in Lipid Bilayer. J. Chem. Theory Comput. 2012, 8, 1200-1211. 
(39) Wennberg, C. L.; van der Spoel, D.; Hub, J. S. Large Influence of Cholesterol on Solute Partitioning Into Lipid Membranes. J. Am. Chem. Soc. 2012, 134, 5351-5361.

(40) Sangster, J. Octanol-Water Partition Coefficients of Simple Organic Compounds. J. Phys. Chem. Ref. Data 1989, 18, 1111-1229.

(41) Machatha, S. G.; Yalkowsky, S. H. Comparison of the Octanol/ Water Partition Coefficients Calculated by ClogP, ACDlogP and KowWin to Experimentally Determined Values. Int. J. Pharm. 2005, 294, 185-192.

(42) Marrink, S. J.; Berendsen, H. Simulation of Water Transport Through a Lipid Membrane. J. Phys. Chem. 1994, 98, 4155-4168.

(43) Pronk, S.; Páll, S.; Schulz, R.; Larsson, P.; Bjelkmar, P.; Apostolov, R.; Shirts, M. R.; Smith, J. C.; Kasson, P. M.; van der Spoel, D.; Hess, B.; Lindahl, E. GROMACS 4.5: a High-Throughput and Highly Parallel Open Source Molecular Simulation Toolkit. Bioinformatics 2013, 29, 845-854.

(44) Abraham, M. J.; Murtola, T.; Schulz, R.; Páll, S.; Smith, J. C.; Hess, B.; Lindahl, E. GROMACS: High Performance Molecular Simulations Through Multi-Level Parallelism From Laptops to Supercomputers. SoftwareX 2015, 1-2, 19-25.

(45) Berger, O.; Edholm, O.; Jähnig, F. Molecular Dynamics Simulations of a Fluid Bilayer of Dipalmitoylphosphatidylcholine at Full Hydration, Constant Pressure, and Constant Temperature. Biophys. J. 1997, 72, 2002-2013.

(46) Schmid, N.; Eichenberger, A. P.; Choutko, A.; Riniker, S.; Winger, M.; Mark, A. E.; Van Gunsteren, W. F. Definition and Testing of the GROMOS Force-Field Versions 54A7 and 54B7. Eur. Biophys. J. 2011, 40, 843-856.

(47) Jämbeck, J. P. M.; Lyubartsev, A. P. Derivation and Systematic Validation of a Refined All-Atom Force Field for Phosphatidylcholine Lipids. J. Phys. Chem. B 2012, 116, 3164-3179.

(48) Darden, T.; York, D.; Pedersen, L. Particle Mesh Ewald: an N. $\log (\mathrm{N})$ Method for Ewald Sums in Large Systems. J. Chem. Phys. 1993, 98, 10089-10092.

(49) Essmann, U.; Perera, L.; Berkowitz, M. L.; Darden, T.; Lee, H.; Pedersen, L. G. A Smooth Particle Mesh Ewald Method. J. Chem. Phys. 1995, 103, 8577-8593.

(50) Hess, B. P-LINCS: a Parallel Linear Constraint Solver for Molecular Simulation. J. Chem. Theory Comput. 2008, 4, 116-122.

(51) Malde, A. K.; Zuo, L.; Breeze, M.; Stroet, M.; Poger, D.; Nair, P. C.; Oostenbrink, C.; Mark, A. E. An Automated Force Field Topology Builder (ATB) and Repository: Version 1.0. J. Chem. Theory Comput. 2011, 7, 4026-4037.

(52) Henchman, R. H.; Essex, J. W. Free Energies of Hydration Using Restrained Electrostatic Potential Derived Charges via Free Energy Perturbations and Linear Response. J. Comput. Chem. 1999, 20, 499-510.

(53) Vanquelef, E.; Simon, S.; Marquant, G.; Garcia, E.; Klimerak, G.; Delepine, J. C.; Cieplak, P.; Dupradeau, F. Y. R.E.D. Server: a Web Service for Deriving RESP and ESP Charges and Building Force Field Libraries for New Molecules and Molecular Fragments. Nucleic Acids Res. 2011, 39, W511-W517.

(54) Jämbeck, J. P. M.; Lyubartsev, A. P. An Extension and Further Validation of an All-Atomistic Force Field for Biological Membranes. J. Chem. Theory Comput. 2012, 8, 2938-2948.

(55) Irwin, J. J.; Shoichet, B. K. ZINC-a Free Database of Commercially Available Compounds for Virtual Screening. J. Chem. Inf. Model. 2005, 45, 177-182.

(56) Berendsen, H.; Postma, J.; Van Gunsteren, W. F.; Di Nola, A.; Haak, J. R. Molecular-Dynamics with Coupling to an External Bath. J. Chem. Phys. 1984, 81, 3684-3690.

(57) Parrinello, M. Polymorphic Transitions in Single Crystals: a New Molecular Dynamics Method. J. Appl. Phys. 1981, 52, 71827190.

(58) Jendrasiak, G. L.; Hasty, J. H. The Hydration of Phospholipids. Biochim. Biophys. Acta, Lipids Lipid Metab. 1974, 337, 79-91. 